INRA Prod. Anim.

1996, 9 (4), 255-264
J.P. LALLÈS, R. TOULLEC

INRA Laboratoire du Jeune Ruminant 65 rue de Saint-Brieuc

35042 Rennes Cedex

\section{Digestion des protéines végétales et hypersensibilité digestive chez le veau préruminant}

La compétitivité de la filière veau de boucherie nécessite de plus en plus de remplacer la poudre de lait écrémé dans les aliments d'allaitement par des produits issus du lactosérum et des concentrats protéiques végétaux. Le soja est actuellement la source végétale la plus employée. Cependant, le choix des dérivés du soja est limité par la forte variabilité de la digestibilité de leurs protéines et par les réactions d'hypersensibilité digestive qu'elles peuvent induire lorsqu'elles sont insuffisamment dénaturées. Il a ainsi été montré que les globulines du soja peuvent échapper en partie à la digestion dans l'intestin grêle. Enfin, les réactions d'hypersensibilité, qui se traduisent notamment par des diarrhées et une baisse de l'ingestion, semblent mettre en jeu des allergènes alimentaires et déclencher des mécanismes immunitaires complexes. La dénaturation des allergènes est essentielle à l'obtention de produits utilisables dans la pratique.

\section{Résumé}

Cet article présente les principaux résultats obtenus récemment sur la digestion des protéines végétales par le veau préruminant, et sur les réactions d'hypersensibilité digestive à médiation immunitaire que leur consommation peut occasionner. Les dosages immunochimiques des activités antigéniques des dérivés du soja doivent être réalisés avec des sérums hyperimmuns plutôt qu'avec des anticorps monoclonaux, et les résultats exprimés quantitativement $(\mathrm{mg}$ d'antigènes $/ \mathrm{g}$ de matières azotées) plutôt que semi-quantitativement (titres). Des équations basées sur des critères analytiques, en particulier le taux de $\beta$-conglycinine et l'activité antitrypsique, permettent de prédire avec précision la digestibilité apparente fécale de l'azote du soja. Lorsque du soja antigénique est consommé, des globulines immunoréactives échappent à la digestion dans l'intestin grêle, parmi lesquelles des polypeptides basiques intacts de la glycinine et des molécules intactes d' $\alpha$-conglycinine.

La plupart des protéines du soja, en particulier la glycinine et la $\beta$-conglycinine, interviennent comme allergènes dans des réactions immunitaires mettant en jeu des anticorps ou des cellules. Les troubles d'hypersécrétion et de motricité intestinales associés sont principalement liés à une libération d'histamine. L'accroissement de la densité des lymphocytes $\mathrm{T}$ auxiliaires (CD4+) dans la lamina propria intestinale et des lymphocytes T suppresseurs-cytotoxiques (CD8+) dans l'épithélium pourrait jouer un rôle important dans les altérations tissulaires de l'intestin, observées lors de la consommation prolongée de soja antigénique.

Parmi les autres protéines végétales, celles de blé et de lupin sont bien digérées et apparemment bien tolérées par le veau.
L'application des quotas laitiers dans l'Union européenne, à partir de 1984, a rendu nécessaire la diversification des sources de protéines dans les aliments d'allaitement; ceci a permis une meilleure adaptation aux variations conjoncturelles de disponibilité et de prix de la poudre de lait écrémé. Après le lactosérum, le soja est la première source de protéines de remplacement, mais son introduction à taux élevé a généralement pour conséquence de réduire de façon variable et difficilement prévisible la digestibilité des aliments et les performances des animaux (revue de Lallès 1993). Ainsi, le tourteau de soja cuit de manière à inactiver suffisamment les facteurs antinutritionnels (antiprotéases, lectines, etc.) considérés comme nocifs pour le porc et les volailles, est très mal utilisé par le veau préruminant. De nouvelles technologies apparues depuis les années 80 et basées sur la dénaturation des protéines en milieu hydroéthanolique à chaud ou par protéolyse, ont permis d'obtenir des produits mieux utilisés par le veau. Les meilleurs d'entre eux peuvent maintenant apporter jusqu'à environ $50 \%$ des 
protéines totales, au lieu de 15 à $20 \%$ antérieurement. Cependant, aucune étude n'avait porté sur l'origine biochimique ou la quantité de protéines alimentaires indigérées ; aucun critère analytique n'avait été défini pour prédire quantitativement la digestibilité de l'azote des dérivés du soja.

Il est maintenant bien connu que des produits du soja insuffisamment traités induisent des désordres digestifs impliquant le système immunitaire, chez les individus prédisposés. Ces troubles se caractérisent par une réduction de l'ingestion et de la croissance, par des diarrhées abondantes associées à une digestion et une absorption incomplètes, et à des perturbations de la motricité et du transit digestifs, par une atrophie villositaire et enfin par la production soutenue d'anticorps circulants dirigés contre certaines protéines du soja (Lallès 1993). Malgré les recherches anglo-saxonnes intenses des années 80 , la nature des constituants alimentaires responsables (ou allergènes), les mécanismes immunitaires sous-jacents et les médiateurs impliqués dans les perturbations étaient toujours mal connus.

L'objet de cet article est de faire le point sur les résultats récemment acquis dans les domaines de la digestion des protéines végétales et de l'hypersensibilité digestive chez le veau, et d'en déduire les applications pratiques.

\section{1 / Traitement des protéines de soja et digestion chez le veau}

Les protéines du soja se répartissent entre globulines et albumines (Guéguen et Cerletti 1994). Les globulines sont des protéines de réserve. Elles comprennent la glycinine, la $\beta$-conglycinine et l' $\alpha$-conglycinine, de masses moléculaires respectives 360,160 et $22 \mathrm{kDa}$ environ. Elles représentent respectivement de 20 à 40 , de 15 à 30 et de 3 à $5 \%$ des protéines totales. La glycinine est constituée de six sous-unités comprenant chacune un polypeptide basique de $20 \mathrm{kDa}$ et un polypeptide acide de $40 \mathrm{kDa}$, liés par un pont disulfure. La $\beta$-conglycinine est une glycoprotéine constituée de deux sous-unités acides et d'une sousunité basique de 53 à $76 \mathrm{kDa}$. L' $\alpha$-conglycinine est monomérique et renferme les inhibiteurs trypsiques de Kunitz. Les albumines rassemblent des protéines ayant généralement un rôle physiologique dans la graine, telles que les inhibiteurs trypsiques de Bowman-Birk, les lectines, la $\beta$-amylase et les lipoxygénases.

Parmi les dérivés du soja incorporés dans les aliments d'allaitement, on distingue classiquement les farines, les concentrats et les isolats, selon leur teneur en matières azotées (environ 50, 65 et $90 \%$ par rapport à la matière sèche). Les farines sont obtenues par décorticage et délipidation des graines. Les concentrats sont préparés en éliminant les oligosides par extraction à l'eau ou à l'aide d'un mélange d'eau et d'alcool. Les isolats sont obtenus par solubilisation des protéines en milieu légèrement alcalin, suivie d'une précipitation en milieu acide. Des traitements supplémentaires très divers (chauffage dans des conditions variables de $\mathrm{pH}$, protéolyse, précipitation, etc.) sont appliqués à ces trois catégories de produits pour dénaturer ou éliminer les lectines et les inhibiteurs de protéases. Bien entendu, ces traitements ont également des effets importants sur les autres protéines (glycinine, $\beta$-conglycinine, etc.). Il est donc important de caractériser in vitro la dénaturation des diverses protéines dans les produits issus du soja, afin de prévoir notamment leur valeur nutritionnelle, ainsi que leur antigénicité et leur allergénicité.

\section{1 / Activités antigéniques et facteurs antinutritionnels du soja}

Un produit est considéré comme antigénique lorsqu'une ou plusieurs de ses protéines peuvent être détectées par un dosage immunochimique spécifique (ELISA, inhibition de l'hémagglutination passive, immunotache, etc.) mettant en jeu des anticorps dirigés contre une forme native ou peu dénaturée de ces molécules. L'activité antigénique a d'abord été exprimée de façon relative (Sissons 1982), ce qui rendait très difficile les comparaisons entre produits et entre laboratoires. Il est préférable d'exprimer les résultats en équivalent protéine native, par référence à une gamme de protéine pure, en milligrammes par gramme de matières azotées totales ou de produit. Nous avons développé des dosages ELISA pour la glycinine, la $\beta$-conglycinine, l' $\alpha$-conglycinine et les lectines du soja, utilisant des sérums hyperimmuns spécifiques (Tukur et al 1993, Lallès et al 1996c). Dans les produits délipidés peu traités, les formes antigéniques de ces protéines représentent environ $40 \%$ des matières azotées (tableau 1). La cuisson, précédée ou non d'une extraction des oligosides à l'eau, réduit ces immunoréactivités de 80 à $90 \%$, tandis que le traitement des produits en milieu hydro-éthanolique à chaud, ou par des enzymes protéolytiques, ou encore par des procédés additionnels non dévoilés par les industriels, peut faire disparaître les activités antigéniques (Lallès et al 1996c). Il faut toutefois remarquer que l'activité antitrypsique mesurée par immunochimie ( $\alpha$-conglycinine) ou par activité biologique (TUI), semble être partiellement résistante à la plupart des traitements. A l'inverse, l'immunoréactivité lectine disparaît quasi-totalement à la cuisson.

\section{2 / Critères analytiques et utilisation du soja par le veau}

Comme cela avait été montré par des travaux plus anciens, la cuisson, précédée ou non d'une extraction à l'eau, génère des produits 
Tableau 1. Traitements technologiques, données analytiques et critères in vivo. Glycinine (Glyc), $\beta$-conglycinine ( $\beta C G)$, $\alpha$-conglycinine ( $\alpha C G)$, activité antitrypsique (TUI), digestibilité apparente de l'azote du soja (DANS, en \%), titre d'anticorps (TA).

\begin{tabular}{|c|c|c|c|c|c|c|c|}
\hline & \multicolumn{3}{|c|}{ Protéine antigénique ${ }^{(1)}$} & \multicolumn{2}{|c|}{ Facteur antinutr. ${ }^{(1)}$} & \multicolumn{2}{|c|}{ Critère in vivo } \\
\hline & Glyc & $\beta C G$ & $\alpha \mathrm{CG}$ & TUI & Lectines & DANS & $\mathrm{TA}$ \\
\hline Délipidation ${ }^{(1)}$ & $(227)$ & $(146)$ & $(25)$ & (119) & (14) & & \\
\hline Cuisson & 12,3 & 17,8 & 21,9 & 11,2 & 1,4 & $59-66$ & 6 à 8 \\
\hline Cuisson + autre traitement ${ }^{(2)}$ & 0,4 & 0 & 4,4 & 5,5 & 0,01 & 76 & 2 à 3 \\
\hline Extraction à l'eau + cuisson & 11,9 & 13,7 & 12,4 & 4,9 & 0,07 & $61-71$ & 3 à 7 \\
\hline Extraction à l'eau + autre traitement ${ }^{(2)}$ & 0,01 & 0 & 2,8 & 2,3 & 0 & 81 & 2 \\
\hline Extraction à l'éthanol & 0 & 0 & 8,8 & 3,9 & 0 & 81 & $<1$ \\
\hline Chauffage à différents $p H$ & 4,8 & 0 & 1,6 & 1,2 & 0 & 84 & $<1$ \\
\hline Protéolyse importante & 0 & 0 & 0 & 2,4 & 0 & $82-90$ & $<1$ \\
\hline
\end{tabular}

(1) Concentrations en \% des valeurs observées pour les produits peu traités. Valeurs entre parenthèses : mg de protéines antigéniques ou de lectines par g de matières azotées (MA), unités internationales de trypsine inhibées (TUI) par mg de MA.

(2) Non décrit par le fabricant.

très peu digestibles (tableau 1) (revue de Sissons 1982). Les veaux ont alors des titres élevés d'anticorps anti-soja dans le plasma. Certains d'entre eux développent des réactions d'hypersensibilité digestive qui se manifestent, notamment, par des diarrhées et des refus d'ingestion. Les problèmes sont plus fréquents lorsque l'incorporation des protéines de soja excède $20 \%$ des protéines du régime. Les traitements technologiques visant à une dénaturation chimique (eau-éthanol à chaud) ou enzymatique (protéolyse) plus poussée des protéines du soja améliorent notablement la digestibilité, tout en réduisant la production d'anticorps spécifiques (Lallès et al 1995c). Les troubles digestifs sont alors beaucoup plus rares et l'incorporation des protéines du soja peut dépasser 50 à $60 \%$ des protéines du régime.

Ces observations nous ont permis d'établir des relations linéaires significatives entre, d'une part, la digestibilité apparente fécale de l'azote du soja et, d'autre part, les proportions de protéines "natives " (solubles dans un tampon carbonate et précipitables à $\mathrm{pH} 4,5$ ), celles de glycinine, d' $\alpha$-conglycinine et de $\beta$-conglycinine immunoréactives, et enfin l'activité antitrypsique (Toullec et al 1994b, Lallès et al 1996c). C'est avec la $\beta$-conglycinine que la relation a été la plus forte (figure 1). L'équation de régression linéaire multiple associant l'activité antitrypsique, l' $\alpha$-conglycinine et les protéines "natives " à la $\beta$-conglycinine, permet de prédire la digestibilité de l'azote avec une grande précision (1\%). La $\beta$ conglycinine a un poids déterminant mais, lorsqu'elle n'est plus détectable, la digestibilité peut être prévue à partir de l'activité antitrypsique seule.

\section{3 / Dosage des protéines antigéniques}

Malgré leur relative complexité, il est crucial d'aborder les problèmes du dosage des protéines antigéniques car il conditionne le choix rationnel des produits à utiliser. En effet, nous venons d'indiquer que les dosages immunochimiques des protéines immunoréactives dans les dérivés du soja permettant, dans nos conditions expérimentales, d'obtenir les meilleures corrélations avec la digestibilité de l'azote sont basés sur l'utilisation, en ELISA, des sérums hyperimmuns dirigés contre les formes natives de ces protéines (Tukur et al 1993, Lallès et al 1996c). En revanche, l'emploi de certains anticorps monoclonaux dirigés contre la glycinine ou la $\beta$-conglycinine (Plumb et al 1994, 1995) four-

Figure 1. Relations entre la digestibilité apparente de l'azote du soja (DANS, en \%) et (a) la teneur en $\beta$-conglycinine ( $\beta C G$ ) ou (b) l'activité antitrypsique (TUI). Relations entre les valeurs de DANS mesurées et prédites $(c, d)$ :

(a) $D A N S=80,1-0,67 \beta C G$

(b, d) DANS = 85,7 - 1,5 TUI (produits

sans $\beta$-conglycinine)

(c) DANS = 85,6 - 1,6 TUI - 0,75 $\beta C G-0,1 P N$

$+2,5 \alpha C G$

$P N$ protéines natives, $\alpha C G \alpha$-conglycinine, $M A$ matières azotées (Lallès et al 1996c, reproduit avec l'aimable autorisation de J. Dairy Sci.).
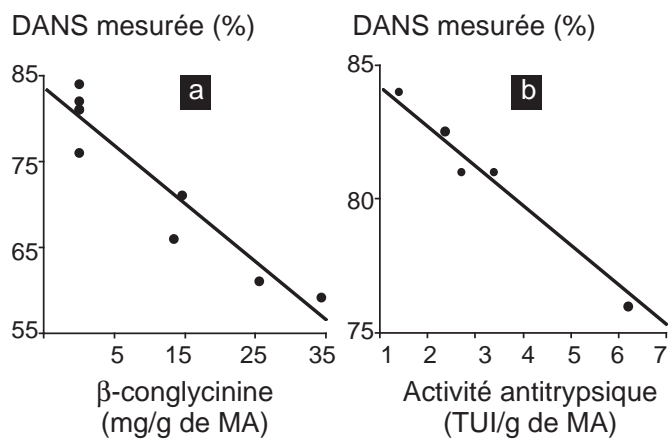

DANS mesurée (\%)

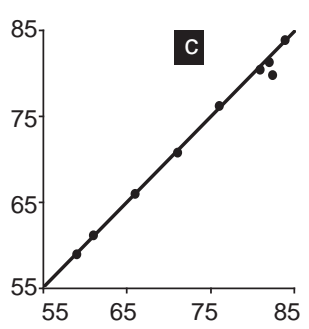

DANS mesurée (\%)

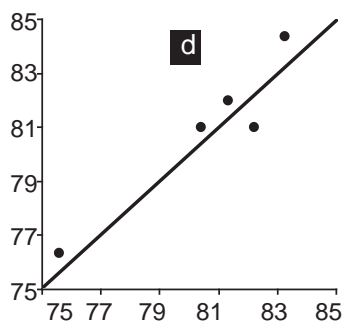

DANS prédite (\%)
Les traitements $\mathrm{du}$ soja visant à la dénaturation poussée des protéines améliorent la digestibilité et réduisent la production d'anticorps. 
nit des activités antigéniques rarement corrélées d'une part à celles obtenues par des sérums hyperimmuns (i.e. " polyclonaux ") spécifiques, et d'autre part aux résultats obtenus in vivo (Lallès et al 1993, thèse de Tukur 1995). Ces anticorps monoclonaux paraissent donc trop spécifiques d'un déterminant antigénique donné pour rendre compte du comportement global du soja in vivo. L'intérêt des anticorps monoclonaux réside plus dans l'étude des modifications de structure des protéines en relation avec les traitements technologiques.

Les dosages ELISA quantitatifs, mis en œuvre par un personnel qualifié et dans des conditions très contrôlées, nécessitent l'emploi de plaques de dosage et de lecteurs spéciaux. Des variantes, basées sur les mêmes principes immunochimiques, mais faisant appel à du matériel plus courant ont été étudiées. Ainsi, les plaques de dosage ELISA sur lesquelles sont fixés les antigènes sont remplacées respectivement par des globules rouges et par des membranes de nitrate de cellulose dans les techniques d'inhibition d'hémagglutination passive et d'immunotache. Quoi qu'il en soit, les corrélations entre les valeurs obtenues par ces diverses techniques sont très fortes, de même que les corrélations avec la digestibilité de l'azote ou le titre d'anticorps (tableau 2). Cependant, l'immunotache donne des valeurs d'activités antigéniques plus élevées que les autres techniques (thèse de Tukur 1995). Dans la pratique, le test d'immunotache est mieux adapté que l'inhibition de l'hémagglutination passive à l'analyse rapide des matières premières par l'utilisateur, l'ELISA quantitatif étant plus contraignant au plan matériel et humain.

\section{4 / Digestion dans l'intestin grêle}

La fin de l'intestin grêle est le site de choix pour étudier l'origine et la quantité des fractions alimentaires ou endogènes indigérées car celles-ci sont remaniées de façon importante par les microbes du gros intestin. La digestibilité apparente moins élevée de l'azote du soja par rapport à celle de l'azote du lait peut être due à la fois à une digestibilité réelle moindre et à une augmentation des pertes d'azote endogène (Sève et Henry 1996). Malheureusement, les mesures directes de ces flux par des techniques d'isotopes stables (azote 15) sont encore rares chez le veau.
Tolman et Beelen (1996) ont toutefois montré que le remplacement de la poudre de lait écrémé par un isolat de soja partiellement hydrolysé entraînait une légère augmentation $(9 \%)$ des pertes de matières azotées endogènes, sans modifier la composition en acides aminés de leur fraction protidique. La digestibilité réelle de l'azote était très voisine de 1 pour le lait et de 0,96 pour le soja. Les quantités de matières azotées endogènes et/ou alimentaires indigérées seraient probablement plus importantes avec des sojas moins élaborés.

Les matières azotées endogènes peuvent être divisées en deux fractions : non spécifique (FEN) et spécifique (FES) (Sève et Henry 1996). FEN provient du fonctionnement intrinsèque du tube digestif ; FES est due aux caractéristiques de la source de protéines alimentaires, telles que les activités antinutritionnelles. Un moyen de pallier en partie la difficulté de la mesure des pertes endogènes totales avec les diverses sources de protéines est de corriger les quantités totales indigérées par soustraction de FEN. La différence représente la somme [(fractions alimentaires + FES) indigérées] = FAES. Puisque la digestibilité réelle des matières azotées du lait est très voisine de 1 , les flux d'azote et d'acides aminés indigérés avec le lait peuvent être considérés comme représentatifs de FEN. Cette approche permet de calculer la digestibilité vraie [(ingéré - FAES) / ingéré], qui fournit des valeurs additives pour les apports d'azote et d'acides aminés par les diverses sources de protéines introduites en mélange dans un aliment. Toutefois, elle ne prend pas en compte la totalité des interactions entre l'aliment et le tube digestif, puisqu'une production endogène accrue entraîne une diminution de l'efficacité de l'utilisation métabolique des acides aminés. Les digestibilités vraies ainsi calculées pour trois dérivés du soja sont reportées dans la figure 2 . De manière inattendue, les valeurs sont voisines pour la farine de soja antigénique et le concentrat traité à l'alcool (89 et $92 \%$ pour l'azote total). Pourtant, la digestibilité vraie (calculée au niveau fécal) pour d'autres lots des mêmes produits commerciaux a été beaucoup plus faible pour la farine que pour le concentrat (70 et $86 \%$ ) (Lallès et al 1995d, Toullec et al 1994a). Par conséquent, la valeur nutritionnelle des produits issus du soja peut varier non seulement avec le procédé de fabrication, mais également à l'intérieur d'un

Tableau 2. Coefficients de corrélation entre données in vivo et détermination de la $\beta$-conglycinine par différentes techniques immunochimiques in vitro (thèse de Tukur 1995).

\begin{tabular}{|l|l|l|l|}
\hline & ELISA & IHP & \multicolumn{1}{|c|}{ IT } \\
\hline Digestibilité apparente de l'azote du régime & $-0,89 * *$ & $-0,89 * *$ & $-0,92^{* * *}$ \\
Digestibilité apparente de l'azote du soja & $-0,90^{* * *}$ & $-0,89 * *$ & $-0,79^{*}$ \\
Gain de poids vif & $-0,66$ & $-0,75^{*}$ & $-0,52$ \\
Titre d'anticorps plasmatiques anti-soja & $+0,89 * *$ & $+0,89 * *$ & $+0,83^{* *}$ \\
\hline
\end{tabular}

ELISA : enzyme-linked immunosorbent assay, IHP : inhibition de l'hémagglutination passive, IT : immunotache.

Significatif à $* \mathrm{P}<0,05,{ }^{* *} \mathrm{P}<0,01, * * * \mathrm{P}<0,001$. 
Figure 2. Digestibilité vraie de quelques protéines végétales à la fin de l'iléon (d'après les données de Caugant et al 1993, Nune do Prado et al 1989, Branco Pardal et al 1995b, R. Toullec résultats non publiés, Tukur et al 1995).

Origine de l'azote alimentaire : (1) produit étudié (50\%), poudre de lait écrémé (50\%) ; (2) produit étudié (34\%), poudre de lait écrémé (66 \%) ; (3) produit étudié (74\%), poudre de lactosérum (26\%).

Digestibilité vraie $(\%)=\{100$ [ingéré - (indigéré total - perte endogène non spécifique)]/ingéré\}, calculée pour le produit étudié seul $(1,2)$ ou pour le mélange produit étudié + lactosérum (3). NAA : azote des acides aminés.
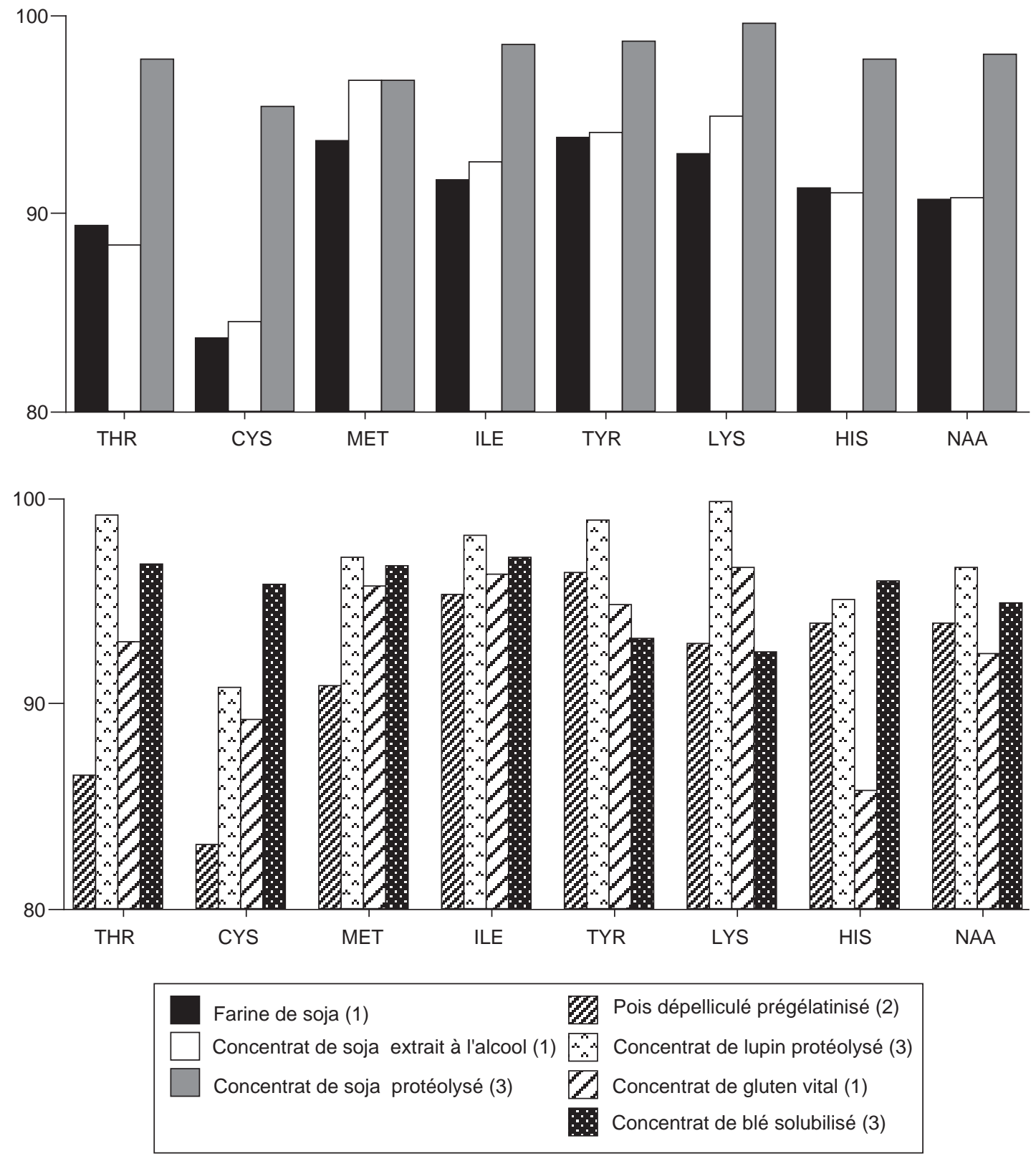

même procédé. En revanche, comme prévu, la digestibilité vraie iléale est beaucoup plus élevée pour le concentrat partiellement protéolysé (98\% pour l'azote total). D'une manière générale, tous les acides aminés indispensables et semi-indispensables, excepté la cystine, présentent des valeurs supérieures ou égales à celles de l'azote total ou sous forme d'acides aminés.

Les fractions azotées indigérées peuvent également être étudiées à l'aide de techniques biochimiques. Nous avons appliqué cette approche à la caractérisation des globulines du soja dans les digesta iléaux. Lorsque l'aliment contient du soja antigénique, les quantités de glycinine, d' $\alpha$-conglycinine et de $\beta$-conglycinine immunoréactives atteignant la fin de l'iléon représentent l'équivalent de 10, 1 et $1 \%$ des quantités respectives ingérées (Tukur et al 1993). En utilisant les techniques d'électrophorèse et d'immuno-empreinte (" Western blotting »), nous avons montré que parmi les entités constitutives de la glycinine, ce sont surtout les polypeptides basiques qui sont retrouvés intacts dans le contenu iléal. Les polypeptides acides sont présents sous forme partiellement digérée, non immunoréactive. De l' $\alpha$-conglycinine intacte est également détectée. Ces techniques immunochimiques ont été inopérantes pour les fractions 
ayant perdu leur immunoréactivité au cours des traitements ou de la digestion. L'utilisation d'anticorps dirigés contre des fractions dénaturées devrait permettre d'étendre ces observations qui demeurent toutefois semiquantitatives. Seul l'emploi des isotopes stables permettra de mesurer les flux de matières azotées alimentaires et endogènes échappant à la digestion dans l'intestin grêle. La glycinine étant moins bien digérée dans l'intestin grêle que la $\beta$-conglycinine, il est surprenant que l'immunoréactivité de cette dernière permette de mieux prédire la digestibilité apparente de l'azote (cf. figure 1). La $\beta$-conglycinine aurait donc un rôle indirect, que nous allons maintenant aborder en examinant les réactions d'hypersensibilité digestive.

\section{2 / Réactions d'hypersensibilité au soja}

\section{1 / Antigènes et allergènes du soja}

Un antigène peut être reconnu par un anticorps produit en réponse à l'injection, à l'ingestion ou encore à l'inhalation de cet antigène. La présence d'anticorps spécifiques indique qu'une molécule est immunogène et qu'un individu est sensibilisé, mais ceci ne conduit pas nécessairement à l'apparition d'une maladie. Un antigène est appelé allergène lorsqu'il provoque des signes cliniques indésirables, postérieurs à la période de sensibilisation, et associés à la présence d'anticorps IgE spécifiques (hypersensibilité immédiate). Toutefois, certaines molécules induisent des réactions pathologiques par des mécanismes immunitaires différents, mettant en jeu des complexes immuns (antigènes-anticorps) insolubles (hypersensibilité semi-retardée), ou des lymphocytes T activés (hypersensibilité retardée).

De nombreuses protéines de soja, parmi lesquelles la glycinine, l' $\alpha$-conglycinine, la $\beta$-conglycinine, les inhibiteurs protéasiques de Bowman-Birk et les lectines, sont immunogènes chez le veau (Lallès et al 1995b). Les réponses immunitaires sont caractérisées notamment par l'abondance des IgG $_{1}$ (Dréau et al 1995, Lallès et al 1995b). L'implication des IgE dans les troubles est suspectée (Sissons 1982), mais leur mise en évidence directe a été très rare (thèse de Dréau 1994). La nature allergénique des protéines de soja n'a donc pas pu être caractérisée par cette propriété. En revanche, l'injection intradermique de protéines purifiées indique que la plupart de celles-ci sont impliquées dans des réactions immunitaires, selon des mécanismes immédiats, semi-retardés ou retardés (Lallès et al 1996b). Ainsi, la réponse cutanée à la glycinine est relativement rapide et brève, alors que celle à la $\beta$-conglycinine persiste au moins cinq jours après injection (figure 3 ). La nature cellulaire de cette dernière réaction est renforcée par le fait que la $\beta$-conglycinine, mais pas la glycinine, induit la prolifération in vitro des lymphocytes sanguins de veaux ayant consommé du soja antigénique (Lallès et al 1996b). Par ailleurs, la $\beta$-conglycinine injectée intradermiquement a un pouvoir inflammatoire local beaucoup plus important que les autres protéines de soja chez les veaux ayant été nourris en permanence au lait. L'ensemble de ces résultats suggère qu'elle pourrait avoir des effets physiopathologiques particuliers. Cela est cohérent avec son poids déterminant dans la prévision de la digestibilité de l'azote.

\section{2 / Altérations morphologiques, cellulaires et fonctionnelles de l'intestin}

Les principales caractéristiques des réactions d'hypersensibilité digestive chez le veau sont une atrophie villositaire partielle, une digestion et une absorption incomplètes, un transit accéléré, une hypermotricité et des diarrhées (Sissons 1982, Lallès 1993). Elles sont associées à des changements cellulaires survenant dans la muqueuse intestinale. Ainsi, les densités de lymphocytes B (tous isotypes) et de lymphocytes T auxiliaires (CD4+) et suppresseurs (CD8+) sont fortement accrues dans la lamina propria, et celle des $\mathrm{T}$ CD8+ dans l'épithélium intestinal (tableau 3). Les lymphocytes spécifiques T CD4+, par le biais des médiateurs qu'ils libèrent (cytokines), pourraient perturber le renouvellement de l'épithélium et stimuler l'activité des muscles lisses sous-jacents. Une cytotoxicité

Figure 3. Réponses cutanées une heure et 5 jours après l'injection intradermique de protéines de soja, chez des veaux ayant consommé pendant 3 mois des régimes lactés à base de poudre de lait écrémé ou de soja antigénique (adapté de Lallès et al 1996b).
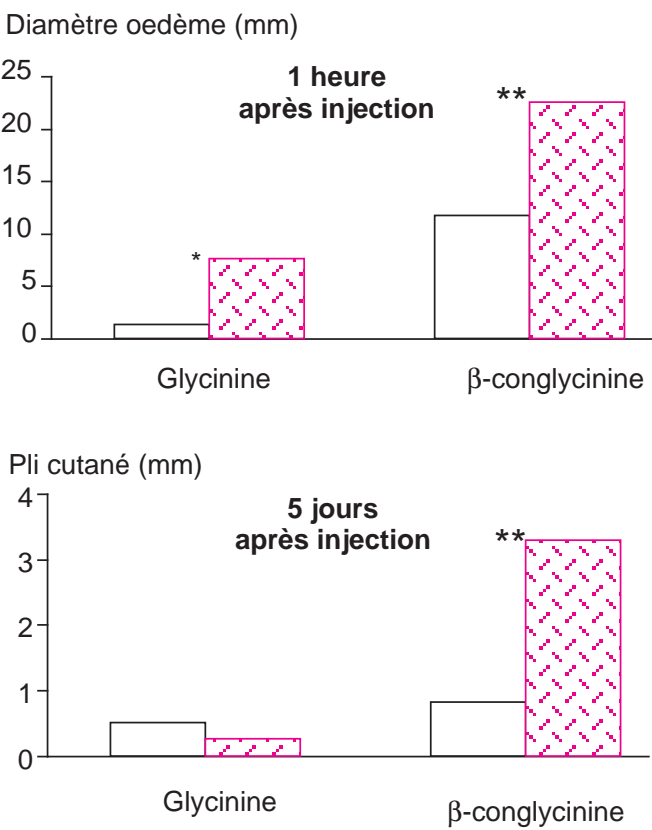

$\square$ témoin $\square$ soja 
Tableau 3. Influence du type de soja incorporé dans l'aliment d'allaitement sur la densité de quelques sous-populations lymphocytaires intestinales (jéjunum) chez le veau préruminant (valeurs en \% de celles observées chez les témoins alimentés au lait) (adapté de Lallès et al 1996a).

\begin{tabular}{|l|c|c|}
\hline Soja & non antigénique & antigénique \\
\hline Lamina propria & & \\
lymphocytes B & $161^{\mathrm{a}}$ & $305^{\mathrm{b}}$ \\
lymphocytes T CD4+ & $130^{\mathrm{a}}$ & $228^{\mathrm{b}}$ \\
lymphocytes T CD8+ & $133^{\mathrm{a}}$ & $279^{\mathrm{b}}$ \\
Epithélium & & \\
lymphocytes T CD4+ & 138 & 400 \\
lymphocytes T CD8+ & $95^{\mathrm{a}}$ & $210^{\mathrm{b}}$ \\
\hline
\end{tabular}

$\mathrm{a}, \mathrm{b} \square$ : différences entre traitements significatives à $\mathrm{P}<0,05$.

des lymphocytes T CD8+ vis-à-vis des entérocytes est également possible. D'autres cellules, telles que les mastocytes intestinaux, armés par des anticorps (IgE ou autres) dirigés contre certaines protéines du soja, participent probablement à l'inflammation locale et aux manifestations cliniques, en libérant des médiateurs. Parmi ceux-ci, l'histamine (via les récepteurs de type $\mathrm{H}-1$ ) joue un rôle important dans les perturbations sécrétoires et motrices (Lallès et al 1994). Ces troubles apparaissent lorsque plus de $20 \%$ des protéines sont apportées par une farine de soja chauffé ayant conservé une forte antigénicité (Lallès et al 1995a), la glycinine et la $\beta$ conglycinine immunoréactives représentant alors 7 et $6 \mathrm{mg}$ par $\mathrm{g}$ de matières azotées ingérées. Lorsque les animaux consomment du soja antigénique en permanence, la perméabilité intestinale est affectée, mais seulement transitoirement (Branco Pardal et al 1995a).

Les principaux mécanismes immunitaires et physiopathologiques de l'hypersensibilité intestinale au soja chez le veau sont schématisés sur la figure 4.

\section{3 / Quelle place pour les autres protéines végétales?}

D'autres sources de protéines, telles que le blé, le pois, le lupin et le maïs, sont utilisables par le veau, après des traitements appropriés.

Les protéines de blé sont disponibles en grande quantité sous forme de concentrats $(80$ $\%$ de matières azotées) préparés industriellement. Le gluten natif (ou vital) est insoluble et se caractérise par sa viscoélasticité. Celle-ci peut être supprimée par chauffage, désamidation chimique de l'asparagine et de la glutamine, ou hydrolyse partielle, les deux derniers traitements permettant d'obtenir des produits solubles. La digestibilité apparente fécale de l'azote des concentrats protéiques de blé est très élevée, bien qu'une légère réduction ait été observée à des taux d'incorporation élevés (tableau 4). Les mesures effectuées à la fin de l'iléon, tant par la comparaison des profils d'acides aminés (Bush et al 1992b, BrancoPardal et al 1995b) que par infusion de leucine marquée à l'azote 15 (Tolman et Beelen 1996), suggèrent que cela résulte de la digestion incomplète de certaines fractions alimentaires plus que d'une augmentation des pertes endogènes. Les fractions alimentaires indigérées pourraient être principalement constituées par le peptide répétitif Pro-Gln-Gln-ProPhe-Pro-Gln, lequel est très abondant dans la partie N-terminale des protéines majeures du gluten (Tatham et Schrewry 1985). La digestibilité vraie est légèrement plus élevée pour les protéines de blé solubilisées par voie chi-

Figure 4. Mécanismes immunitaires et physiopathologiques de l'hypersensibilité intestinale au soja chez le veau.

L'activité pro-inflammatoire putative de la $\beta$-conglycinine (1) augmenterait la perméabilité de l'intestin aux protéines alimentaires (2), induisant une réponse immunitaire locale (3) conduisant à la sensibilisation.

Il y aurait émergence, dans la lamina propria, de lymphocytes $T$ auxiliaires (CD4) spécifiques de l'aliment. Ceux-ci contrôlent l'ensemble de la réponse immunitaire, qui comprend des lymphocytes $T$ cytotoxiques (CD8), des lymphocytes B spécifiques, et diverses autres cellules (mastocytes: MC; cellules présentatrices de l'antigène : APC ; etc.). L'atrophie villositaire pourrait être la conséquence d'une stimulation des centres germinatifs entérocytaires par des cytokines (ctk) et/ou d'une destruction de l'épithélium par cytotoxicité impliquant des lymphocytes T CD8. L'hyperactivité motrice et l'hypersécrétion intestinales résulteraient de l'action de l'histamine, probablement libérée par les mastocytes armés par des IgE spécifiques des allergènes alimentaires.

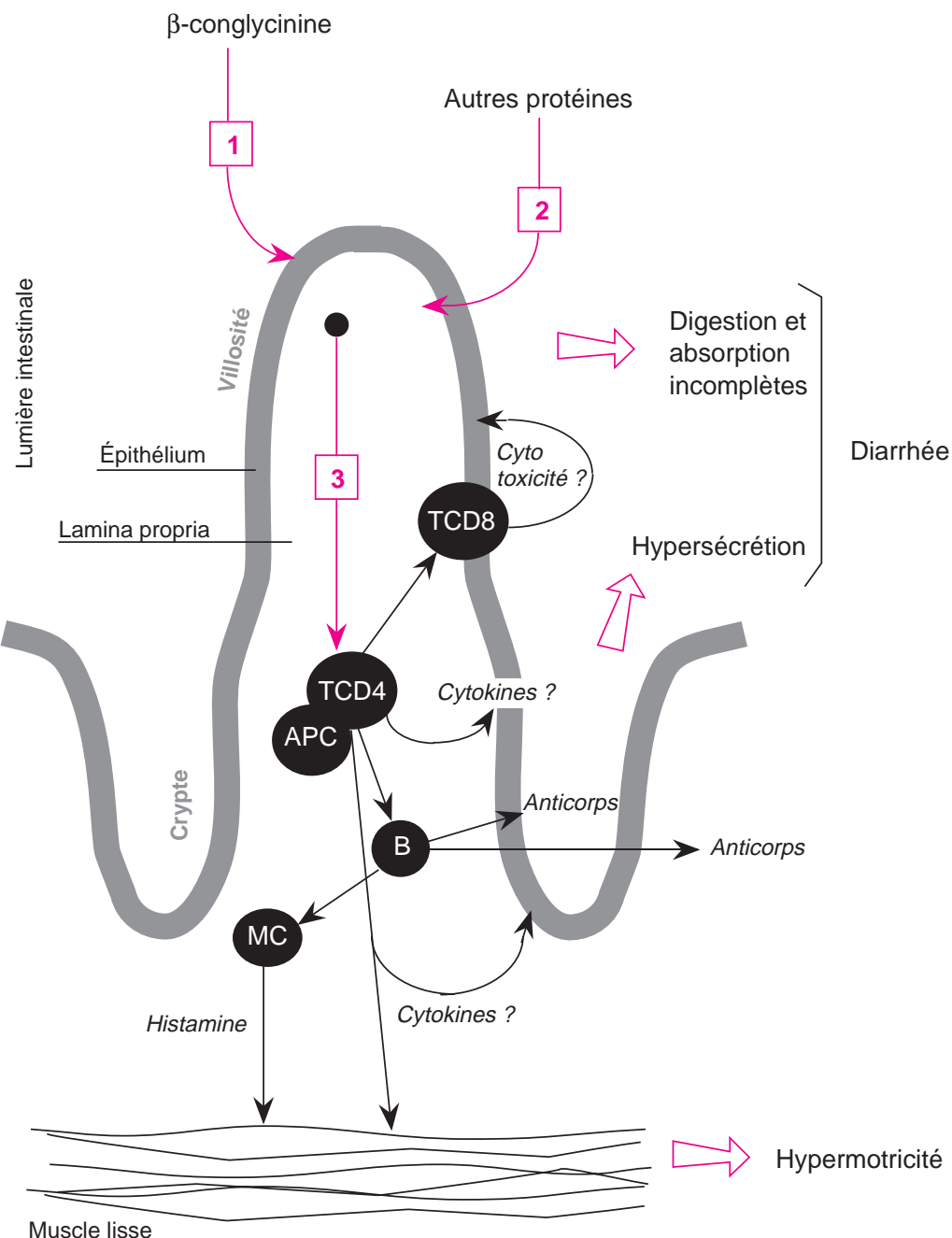


mique que pour le gluten natif (95\% vs 92 pour l'azote sous forme d'acides aminés) (cf. figure 2).

La forte teneur en amidon et en $\alpha$-galactosides du pois limite son incorporation en l'état dans les aliments d'allaitement. La fabrication de concentrats ou d'isolats protéiques n'a pas encore vraiment atteint le développement industriel que l'on connaît dans le cas des protéines de soja ou même de blé. Aussi, la farine de pois nécessite-t'elle d'être floconnée (Toullec et al 1992) ou prégélatinisée pour que sa digestibilité soit élevée (tableau 4). En effet, la farine crue conduit à une sensibilisation rapide des veaux et à une réduction importante de la digestibilité apparente iléale (Bush et al 1992a). Ceci correspond essentiellement à une augmentation des pertes d'azote endogène, bien qu'une partie de la légumine du pois (analogue de la glycinine du soja) échappe également à la digestion intestinale. La digestibilité vraie du pois prégélatinisé est voisine de celle du gluten de blé vital, excepté pour la thréonine et les acides aminés soufrés d'une part, et pour l'histidine d'autre part, qui présentent des valeurs respectivement plus faibles et plus élevées avec le pois (cf. figure 2). Sa digestibilité est moins élevée au niveau fécal qu'à la fin de l'iléon (tableau 4), probablement parce qu'une part importante de l'amidon échappe à la digestion dans l'intestin grêle, favorisant ainsi la prolifération microbienne dans le gros intestin. Les protéines de lupin, sous forme de concentrat partiellement protéolysé, sont très digestibles et non immunogènes ou allergènes (Tukur et al 1995). Les protéines de maïs, introduites sous forme de concentrat, sont également bien digérées ; elles ne semblent pas provoquer de réactions d'hypersensibilité malgré leur immunogénicité notable (Toullec et Grongnet 1990).

\section{Conclusions et perspectives}

L'activité antigénique des dérivés du soja doit être déterminée par des tests immunochimiques quantitatifs basés sur l'emploi de sérums hyperimmuns dirigés contre les antigènes natifs les plus importants, principalement la $\beta$-conglycinine. Le test d'immunotache est bien adapté à l'analyse rapide des matières premières par l'utilisateur ; l'ELISA quantitatif nécessite des conditions de laboratoire mieux contrôlées et un personnel expérimenté. Ces résultats, associés à l'activité antitrypsique, permettent alors d'estimer de façon fiable la digestibilité fécale apparente de l'azote du soja par le veau. Les dérivés du soja sont d'autant mieux digérés et moins immunogènes que leurs activités antigéniques sont faibles in vitro. Les meilleurs produits peuvent alors apporter jusqu'à $50 \%$ des protéines $\mathrm{du}$ régime sans trop altérer les performances des animaux. Il faut toutefois respecter toutes les autres contraintes de formulation. Lorsque du soja antigénique est consommé, des petites quantités de protéines alimentaires, en particulier les polypeptides basiques de la glycinine et l' $\alpha$-conglycinine, atteignent la fin de l'intestin grêle en ayant été très peu digérées et sous forme immunoréactive.

Les réactions d'hypersensibilité digestives observées avec des produits fortement antigéniques mettent en jeu des réactions immunitaires complexes qui semblent impliquer fortement la $\beta$-conglycinine, des lymphocytes $\mathrm{T}$ spécifiques, et probablement d'autres cellules à l'origine de la libération de médiateurs tels que l'histamine. La démonstration de l'implication de la $\beta$-conglycinine dans les troubles digestifs permettra de valider l'intérêt pratique du dosage immunochimique de cette

Tableau 4. Digestibilité apparente fécale et iléale de l'azote, pour différentes sources de protéines incorporées dans les aliments d'allaitement pour veaux.

\begin{tabular}{|c|c|c|c|c|}
\hline \multirow{2}{*}{ Source de protéines } & \multirow{2}{*}{$\begin{array}{l}\text { Taux d'incor- } \\
\text { poration }^{(1)}\end{array}$} & \multicolumn{2}{|c|}{ Digestibilité apparente $\square^{(2)}$} & \multirow{2}{*}{ Référence } \\
\hline & & Fécale & Iléale & \\
\hline \multicolumn{5}{|l|}{ Blé } \\
\hline gluten, vital & 19 & 99 & - & Toullec et Grongnet 1990 \\
\hline gluten, dévitalisé & 19 & 98 & - & Toullec et Grongnet 1990 \\
\hline gluten, vital & 52 & 95 & 92 & Branco Pardal et al 1995b \\
\hline gluten, hydrolysé & 37,5 & 97 & - & R. Toullec, non publié \\
\hline gluten, hydrolysé & 75 & 86 & 92 & $\begin{array}{l}\text { Bush et al } 1992 b \text {, } \\
\text { R. Toullec, non publié }\end{array}$ \\
\hline protéines, solubilisées & 37,5 & - & 94 & R. Toullec, non publié \\
\hline protéines, solubilisées & 75 & - & 92 & R. Toullec, non publié \\
\hline \multicolumn{5}{|l|}{ Pois } \\
\hline dépelliculé, cru (semaine 1) & 34 & 76 & 89 & Bush et al 1992a \\
\hline dépelliculé, cru (semaine 4) & 34 & 70 & 56 & Bush et al 1992a \\
\hline dépelliculé, prégélatinisé & 34 & 84 & 92 & Nunes do Prado et al 1989 \\
\hline Lupin, concentrat protéique & & & & \\
\hline partiellement protéolysé & 71 & 91 & 97 & Tukur et al 1995 \\
\hline Maïs, concentrat protéique & 19 & 91 & - & Toullec et Grongnet 1990 \\
\hline
\end{tabular}

(1) Dans les aliments d'allaitement (\% des matières azotées totales).

(2) Exprimée en \% des régimes témoins à base de poudre de lait écrémé ou de poudre de lait écrémé et de lactosérum. 
protéine dans les dérivés du soja destinés à l'alimentation lactée du veau.

L'utilisation de l'azote 15 pour mesurer les flux de matières azotées alimentaires et endogènes le long de l'intestin grêle devrait permettre de préciser la digestion des protéines alimentaires et les effets des aliments sur le fonctionnement du tube digestif. Les techniques biochimiques permettront, de façon complémentaire, de mieux caractériser la nature et l'importance des fractions indigérées.

\section{Remerciements}

La plupart des travaux ayant servi à l'élaboration de ce texte ont bénéficié du soutien financier de la Direction Générale de l'Enseignement et de la Recherche du Ministère de l'Agriculture et de la Pêche, et de la Région Bretagne, du Département d'Ille-et-Vilaine et du District de Rennes.

\section{Références bibliographiques}

Branco Pardal P., Lallès J.P, André F, Delval E., Toullec R., 1995a. Assessment of gastrointestinal permeability to small marker probes in the preruminant calf. Reprod. Nutr. Develop., 35, 189-200.

Branco-Pardal P., Lallès J.P., Formal M., Guilloteau P., 1995b. Digestion of wheat gluten and potato protein by the preruminant calf : digestibility, amino acid and immunoreactive proteins in ileal digesta. Reprod. Nutr. Develop., 35, 639-654.

Bush R.S., Toullec R., Caugant I., Guilloteau P., 1992a. Effects of raw pea flour on nutrient digestibility and immune responses in the preruminant calf. J. Dairy Sci., 75, 3539-3552.

Bush R.S., Toullec R., Guilloteau P., Barré P., 1992b. Digestibilité iléale d'un gluten de blé partiellement hydrolysé chez le veau préruminant. Ann. Zootech., 41, 31-32.

Caugant I., Toullec R., Formal M., Guilloteau P., Savoie L., 1993. Digestibility and amino acid composition of digesta at the end of the ileum in preruminant calves fed soyabean protein. Reprod. Nutr. Develop., 33, 335-347.

Dréau D., Lallès J.P., Salmon H., Toullec R., 1995.

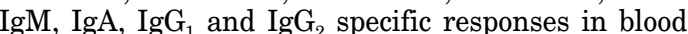
and gut secretion of calves fed soyabean products. Vet. Immunol. Immunopathol., 47, 57-67.

Guéguen J., Cerletti P., 1994. Proteins of some legume seeds : soybean, pea, fababean and lupin. In : B.J.F. Hudson (ed), New and Developing Sources of Food Proteins, 145-193. Chapman \& Hall.

Lallès J.P., 1993. Nutritional and antinutritional aspects of soyabean and field pea proteins used in veal calf production : a review. Livest. Prod. Sci., 34, 181-202.

Lallès J.P., Plumb J.W., Mills E.N.C., Morgan M.R.A., Tukur H.M., Toullec R., 1993. Antigenic activity of some soyabean products used in veal calf feeding : comparison between in vitro tests (ELISA polyclonal vs monoclonal) and with in vivo data. In : A.F.B. van der Poel, J. Huisman, H.S. Saini (eds), Recent Advances of Research in Antinutritional Factors in Legume Seeds, 281-286. Wageningen Pers, The Netherlands.

Lallès J.P., Duvaux-Ponter C., Sissons J.W., Toullec R., 1994. Gut motility disorders in calves sensitive to soya when fed chronically. Neurogastroenterol. Motility, 6,140 .

Lallès J.P., Benkredda D., Toullec R., 1995a. Influence of soya antigen levels in milk replacers on the disruption of intestinal motility patterns in calves sensitive to soya. J. Vet. Med. A, 42, 467-478.
Lallès J.P., Dréau D., Huet A., Toullec R., 1995b. Systemic and local (gut) specific antibody responses in preruminant calves sensitive to soya. Res. Vet. Sci., 59, 56-60.

Lallès J.P., Toullec R., Bouchez P., Roger L., 1995c. Antigenicity and digestive utilization of four soyabean products by the preruminant calf. Livest. Prod. Sci., 41, 29-38.

Lallès J.P., Toullec R., Branco Pardal P., Sissons J.W., 1995d. Hydrolyzed soy protein isolate sustains high nutritional performance in veal calves. J. Dairy Sci., 78, 194-204.

Lallès J.P., Dréau D., Féménia F., Parodi A.L., Toullec R., 1996a. Feeding heated soyabean flour increases the density of $\mathrm{B}$ and $\mathrm{T}$ lymphocytes in the small intestine of preruminant calves. Vet. Immunol. Immunopathol., 52,105-115.

Lallès J.P., Dréau D., Salmon H., Toullec R., 1996b. Identification of soya bean allergens and immune mechanisms of dietary sensitivities in preruminant calves. Res. Vet. Sci., 60, 111-116.

Lallès J.P., Tukur H.M., Toullec R., Miller B.G., 1996c. Analytical criteria for predicting apparent digestibility of soy protein in preruminant calves. J. Dairy Sci., 79, 475-484.

Nunes Do Prado I., Toullec R., Guilloteau P., Guéguen J., 1989. Digestion des protéines de pois et de soja chez le veau préruminant. II - Digestibilité apparente à la fin de l'iléon et du tube digestif. Reprod. Nutr. Develop., 29, 425-439.

Plumb G.W., Mills E.N.C., Tatton M.J., D'Ursel C.C.M., Lambert N., Morgan M.R.A., 1994. Effect of thermal and proteolytic processing on glycinin, the 11 S globulin of soy (Glycine max.) : a study utilizing monoclonal and polyclonal antibodies. J. Agric. Food Sci., $42,834-840$.

Plumb G.W., Lambert N., Mills E.N.C., Tatton M.J., D’Ursel C.C.M., Bogracheva T., Morgan M.R.A., 1995. Characterisation of monoclonal antibodies against $\beta$-conglycinin from soy bean (Glycine max.) and their use as probes for thermal denaturation. J. Sci. Food Agric., 67, 511-520.

Sève B., Henry Y., 1996. Protein utilization in non ruminants. In : A.F. Nunes, A.V. Portugal, J.P. Costa, J.R. Ribeiro (eds), Protein Metabolism and Nutrition, 59-82. E.A.A.P. Publication n ${ }^{\circ} 81$, Santarém, Portugal.

Sissons J.W., 1982. Effects of soyabean products on digestive processes in the gastrointestinal tract of preruminant calves. Proc. Nutr. Soc., 41, 53-61. 
Tatham A.S., Schrewry P.R., 1985. The conformation of wheat gluten proteins. The secondary structures and thermal stabilities of $\alpha, \beta, \gamma$ and $\omega$ gliadins. J. Cereal Sci., 3, 103-113.

Tolman G.H., Beelen G.M., 1996. Endogenous nitrogen and amino acid flow in the terminal ileum of veal calves and true digestibility of skim milk, soluble wheat and soya isolate proteins. In : Veal perspectives to the year 2000, 191-207. Fédération de la vitellerie française, Paris.

Toullec R., Grongnet J.F., 1990. Remplacement partiel des protéines du lait par celles du blé ou du maïs dans les aliments d'allaitement : influence sur l'utilisation digestive chez le veau de boucherie. INRA Prod. Anim., 3, 201-206.

Toullec R., Lallès J.P., Guilloteau P., 1992. Influence $\mathrm{du}$ floconnage sur l'utilisation de la farine de pois dans l'alimentation du veau de boucherie. First European Conference on Grain Legumes, Angers, France. 497-498.
Toullec R., Lallès J.P., Bouchez P., 1994a. Substitution of skim milk protein with soyabean protein concentrates and whey in milk replacers for veal calves. Anim. Feed Sci. Technol., 50, 101-112.

Toullec R., Lallès J.P., Tukur H.M., 1994b. Caractéristiques biochimiques et digestibilité apparente des matières azotées du soja chez le veau préruminant. Renc. Rech. Ruminants, 1, 229-232.

Tukur H.M., Lallès J.P., Mathis C., Caugant I., Toullec R., 1993. Digestion of soybean globulins, glycinin, $\alpha$-conglycinin and $\beta$-conglycinin, in the preruminant and the ruminant calf. Can. J. Anim. Sci., 73, 891905 .

Tukur H.M., Branco Pardal P., Formal M., Toullec R., Lallès J.P., Guilloteau P., 1995. Digestibility, blood levels of nutrients and skin responses of calves fed soyabean and lupin proteins. Reprod. Nutr. Develop., 35, 27-44.

\begin{abstract}
Digestion of plant proteins and gut hypersensitivity in the preruminant calf.

The recent developments of studies on digestion of plant protein sources by the preruminant calf, and on immune-mediated gut hypersensitivity reactions are reviewed. The immuno-chemical determination of the antigenic activity of soyabean products should involve hyperimmune sera rather than monoclonal antibodies, and the data should be expressed quantitatively (mg antigen I g crude protein). Equations based on analytical criteria, particularly $\beta$-conglycinin levels and antitryptic activities, have been set up ; they could accurately predict the faecal digestibility of soyabean nitrogen in vivo. When antigenic soya was consumed, substantial amounts of immunoreactive soyabean globulins escaped small intestinal digestion, of which intact basic polypeptides of glycinin and intact $\alpha$-conglycinin.
\end{abstract}

Abstract
Most soyabean antinutritional factors, including glycinin and $\beta$-conglycinin, were shown to be implicated as allergens in both antibody - and cell-mediated reactions in sensitive calves. Intestinal hyper-secretion and motor disorders associated with these adverse reactions were mediated mainly by histamine release. Increased densities of helper (CD4+) $T$ lymphocytes in the lamina propria and of suppressor-cytotoxic (CD8+) T lymphocytes in the epithelium may probably account for intestinal damage due to chronic antigenic soya consumption.

Other plant protein sources, in particular wheat and lupin, proved to be well digested and tolerated by calves.

LALLÈS J.P., TOULLEC R., 1996. Digestion des protéines végétales et hypersensibilité digestive chez le veau préruminant. INRA Prod. Anim., 9 (4), 255264. 